\title{
GLOBAL ASYMPTOTIC STABILITY OF A PREDATOR-PREY SYSTEM OF HOLLING TYPE
}

AUTHOR(S):

SUGIE, JITSURO; KATAYAMA, MASAKI

CITATION:

SUGIE, JITSURO ... [et al]. GLOBAL ASYMPTOTIC STABILITY OF A PREDATOR-PREY SYSTEM OF HOLLING TYPE. 数理解析研究所講究録 1998, 1034: 142-158

ISSUE DATE:

1998-04

URL:

http://hdl.handle.net/2433/61905

RIGHT: 


\title{
GLOBAL ASYMPTOTIC STABILITY OF A PREDATOR-PREY SYSTEM OF HOLLING TYPE
}

\author{
島根大学 総合理工学部 杉江 実郎 (JITSURO SUGIE) \\ 島根大学 総合理工学部 片山 公貴 (MASAKI KATAYAMA)
}

\section{INTRODUCTION}

We consider a class of predator-prey models of the form

$$
\begin{aligned}
& \dot{x}=r x\left(1-\frac{x}{k}\right)-\frac{x^{p} y}{a+x^{p}}, \\
& \dot{y}=y\left(\frac{\mu x^{p}}{a+x^{p}}-D\right), \\
& x(0)>0, \quad y(0)>0,
\end{aligned}
$$

where $=d / d t$, and where $x(t)$ and $y(t)$ are the densities of the prey and predator, respectively, at given time $t \geq 0$. The parameters $r, k, a, \mu, D$, and $p$ are positive real numbers. The function $\frac{x^{p}}{a+x^{p}}$ in (1.1) represents a functional response of predator to prey. The functional response is said to belong to Holling type II if $p \leq 1$; to Holling type III if $p>1$. The functional response of Holling type is strictly increasing and bounded; if $p \leq 1$, then it is upwards convex; otherwise, it has a inflection point, that is, the functional response curve is sigmoid. This predator-prey model has been widely studied in many papers (for instance, [1]-[10]). Also, we can find this system as an important example in the literature [11]-[17] concerning a generalization of (1.1) which was proposed by Gause [18]

$$
\begin{aligned}
& \dot{x}=x \rho(x)-y \phi(x), \\
& \dot{y}=y(-\gamma+\psi(x)) .
\end{aligned}
$$

System (1.1) has two equilibria $E_{0}(0,0)$ and $E_{1}(k, 0)$. In case

$$
\mu>D \quad \text { and } \quad k>\lambda_{p} \stackrel{\text { def }}{=} \sqrt[p]{\frac{a D}{\mu-D}},
$$

the third equilibrium $E^{*}\left(\lambda_{p}, \nu_{p}\right)$ appears in the region $\{(x, y): x>0$ and $y>0\}$, where

$$
\nu_{p}=\frac{r \mu}{D}\left(1-\frac{\lambda_{p}}{k}\right) \lambda_{p}
$$


The aim of this paper is to present a necessary and sufficient condition under which the positive equilibrium $E^{*}$ of (1.1) is globally asymptotically stable. We say that the positive equilibrium $E^{*}$ is globally aymptotically stable if $E^{*}$ is stable and if every solution of (1.1) tends to $E^{*}$.

Generally speaking, if

(i) all solutions are bounded in the future,

(ii) a unique equilibrium exists and is asymptotically stable,

(iii) no closed orbits exist,

then, by the Poincaré-Bendixson theorem, the unique equilibrium is globally asymptotically stable.

It is easy to show that all solutions of (1.1) and (1.2) are bounded in the future and remain in the region $\{(x, y): x>0$ and $y>0\}$. It is also well known that under the assumptions which ensure that system (1.2) has a unique positive equilibrium,

$$
\left.\frac{d}{d x}\left(\frac{x \rho(x)}{\phi(x)}\right)\right|_{x=x^{*}}<0
$$

implies that the positive equilibrium is (locally) asymptotically stable, where $x^{*}$ is the $x$ coordinate of the positive equilibrium (for example, see [13], [15], [19]). In system (1.1), condition (1.4) coincides with

$$
(p D-(p-1) \mu) k<(p D-(p-2) \mu) \lambda_{p}
$$

If assumption (1.3) fails, then no positive equilibrium exists and, therefore, system (1.1) has no closed orbits. Recently Sugie, Kohno and Miyazaki [10] discussed the case that the positive equilibrium $E^{*}$ exists and gave the following sufficient condition for the nonexistence of closed orbits of (1.1).

Theorem A ([10]). Let $p$ be a positive number with $p \leq \frac{1}{2}$ or $p \geq 1$. If (1.3) and

$$
(p D-(p-1) \mu) k \leq(p D-(p-2) \mu) \lambda_{p}
$$

are satisfied, then system (1.1) has no closed orbits.

By virtue of Theorem A, we see that if (1.3) and (1.5) hold, then the positive equilibrium $E^{*}$ of $(1.1)$ is globally asymptotically stable when $p \leq \frac{1}{2}$ or $p \geq 1$. However, the case

$$
(p D-(p-1) \mu) k=(p D-(p-2) \mu) \lambda_{p}
$$

is delicate. To answer this delicate problem, we need to examine the behavior of trajectories near the positive equilibrium $E^{*}$ of (1.1).

A trajectory is said to be a homoclinic orbits if its $\alpha$ - and $\omega$-limit sets are the origin. If system (1.1) has a homoclinic orbit, then the positive equilibrium $E^{*}$ is not even stable. In 
Section 2 we show that system (1.1) has no homoclinic orbits. Hence, it follows form (i) that every positive semitrajectory of (1.1) keeps on rotating around the positive equilibrium $E^{*}$, in counterclockwise order; or ultimately, it approaches $E^{*}$ without rotating around $E^{*}$. Moreover, by Theorem $\mathrm{A}$ and (iii), we see that the positive equilibrium $E^{*}$ of (1.1) is also globally asymptotically stable in the critical case (1.7).

In Section 3 we lift the restriction that $p \leq \frac{1}{2}$ or $p \geq 1$. To be more exact, we consider the case $0<p<1$ and prove that if (1.6) is satisfied, then system (1.1) has no closed orbits.

In Section 4 we prove the main result of this paper:

Theorem 1.1. Assume (1.3). Then the positive equilibrium $E^{*}$ of (1.1) is globally asymptotically stable if and only if (1.6) is satisfied.

\section{NON-EXISTENCE OF HOMOCLINIC ORBITS}

We first examine the asymptotic behavior of trajectories in a neighborhood of the origin of the Liénard system

$$
\begin{aligned}
& \frac{d u}{d \tau}=v-F(u), \\
& \frac{d v}{d \tau}=-g(u),
\end{aligned}
$$

where $F(u)$ and $g(u)$ are continuously differentiable and

$$
F(0)=0 \quad \text { and } \quad u g(u)>0 \quad \text { if } u \neq 0 .
$$

In particular, we concentrate our attention on the problem when system (2.1) has homoclinic orbits. Taking account of the vector field of (2.1) and assumption (2.2), we see that

(i) if there exists a homoclinic orbit of (2.1), then the origin is not stable,

(ii) if system (2.1) has a homoclinic orbit, then all trajectories of (2.1) in the region that is enclosed by the union of the homoclinic orbit and the origin are also homoclinic orbits,

(iii) if a homoclinic orbit exists in the upper half-plane $\{(u, v): u>0$ and $v \in \mathbf{R}\}$ (resp., the lower half-plane $\{(u, v): u<0$ and $v \in \mathbf{R}\}$ ), then other homoclinic orbits exist in the upper (resp., lower) half-plane.

This problem resolves itself into the question whether the positive semitrajectory of (2.1) starting at any point on the vertical isocline $\{(u, v): u \in \mathbf{R}$ and $v=F(u)\}$ crosses the $y$-axis at some finite time or approaches the origin without intersecting the $x$-axis. Sugie and Hara [20] discussed the question in detail and gave some sufficient conditions for the non-existence of homoclinic orbits of (2.1). For the sake of convenience, we denote

$$
G(x)=\int_{0}^{x} g(\sigma) d \sigma
$$


$C^{+}=\{(x, y): x>0$ and $y=F(x)\} \quad$ and $\quad C^{-}=\{(x, y): x<0$ and $y=F(x)\}$.

THEOREM B ([20]). Suppose that

$$
F(x) \leq 2 \sqrt{2 G(x)}-h(\sqrt{2 G(x)})
$$

for $x>0$ (resp., $x<0$ ), $|x|$ sufficiently small, where $h(\sigma)$ is a non-negative continuous function with

$$
\begin{aligned}
& \frac{h(\sigma)}{\sigma} \text {, is non-decreasing and is not greater than } 2 \\
& \text { for } \sigma>0 \text { sufficiently small, }
\end{aligned}
$$

$$
\int_{0}^{\sigma_{0}} \frac{h(\sigma)}{\sigma^{2}} d \sigma=\infty \quad \text { for some } \sigma_{0}>0
$$

Then the positive (resp., negative) semitrajectory of (2.1) passing through any point on the curve $C^{+}\left(\right.$resp., $\left.C^{-}\right)$meets the negative $y$-axis and, therefore, system (2.1) has no homoclinic orbits in the upper half-plane.

THEOREM C ([20]). Suppose that

$$
F(x) \geq-2 \sqrt{2 G(x)}+h(\sqrt{2 G(x)})
$$

for $x>0$ (resp., $x<0$ ), $|x|$ sufficiently small, where $h(\sigma)$ is a non-negative continuous function satisfying (2.4) and (2.5). Then the negative (resp., positive) semitrajectory of (2.1) passing through any point on the curve $C^{+}$(resp., $C^{-}$) meets the positive $y$-axis and, therefore, system (2.1) has no homoclinic orbits in the lower half-plane.

Let $h(\sigma)=\sigma$. Then $h(\sigma)$ satisfy conditions (2.4) and (2.5). For simplicity, let ' $=d / d u$. Suppose that $F^{\prime}(0)<0$. Then, by $(2.2)$ we have

$$
F(x)<0<\sqrt{2 G(x)}=2 \sqrt{2 G(x)}-h(\sqrt{2 G(x)})
$$

for $x>0$ sufficiently small, and

$$
F(x)>0>-\sqrt{2 G(x)}=-2 \sqrt{2 G(x)}+h(\sqrt{2 G(x)})
$$

for $x<0,|x|$ sufficiently small. Hence, conditions (2.3) and (2.6) are also satisfied for $x>0$ and $x<0$, respectively. Thus, from Theorems B and C, we see that system (2.1) has no homoclinic orbits. Similarly, if $F^{\prime}(0)>0$, then system (2.1) has no homoclinic orbits.

We consider the case that $F^{\prime}(0)=0$. If $g^{\prime}(0)>0$, then there exists an $\varepsilon_{0}>0$ such that

$$
\sqrt{2 G(x)}>\varepsilon_{0}|x|
$$

for $|x|>0$ small enough. Hence, we have

$$
|F(x)|<\varepsilon_{0}|x|<\sqrt{2 G(x)}=2 \sqrt{2 G(x)}-h(\sqrt{2 G(x)})
$$


for $|x|>0$ small enough and, therefore, conditions (2.3) and (2.6) hold for both $x>0$ and $x<0$. Thus, system (2.1) has no homoclinic orbits. From Theorems B and C we also see that all positive semitrajectories of (2.1) near the origin keep on rotating around the origin in this case.

To sum up, we have the following result.

THEOREM 2.1. If $F^{\prime}(0) \neq 0$, then system (2.1) has no homoclinic orbits; if $F^{\prime}(0)=0$ and $g^{\prime}(0)>0$, then all positive semitrajectories of (2.1) near the origin keep on rotating around the origin and, therefore, system (2.1) has no homoclinic orbits.

Let us now return to the Gause predator-prey model (1.2). We assume that the functions in system (1.2) are sufficiently smooth on $[0, \infty)$ and satisfy the following:

(i) there exists a $K>0$ such that $(x-K) \rho(x)<0$ if $x \neq K$,

(ii) $\phi(0)=\psi(0)=0$ and $\phi^{\prime}(x)>0$ and $\psi^{\prime}(x)>0$ for $x>0$,

(iii) there exists an $x^{*}$ with $0<x^{*}<K$ such that $\psi\left(x^{*}\right)=\gamma$.

Put $y^{*}=\frac{x^{*} \rho\left(x^{*}\right)}{\phi\left(x^{*}\right)}$. Then system (1.2) has a unique positive equilibrium $\left(x^{*}, y^{*}\right)$.

For the sake of convenience, we define

$$
\Phi(x)=\int_{x^{*}}^{x} \frac{\phi^{\prime}(\sigma)}{\phi(\sigma)} d \sigma .
$$

Then we can transform the Gause-type model (1.2) into system (2.1) with

$$
\begin{gathered}
F(u)=\int_{0}^{u}\left\{\left(-\gamma+\psi\left(\sigma+x^{*}\right)\right)+\phi\left(\sigma+x^{*}\right) \frac{d}{d \sigma}\left(\frac{\left(\sigma+x^{*}\right) \rho\left(\sigma+x^{*}\right)}{\phi\left(\sigma+x^{*}\right)}\right)\right\} \exp \left\{-\Phi\left(\sigma+x^{*}\right)\right\} d \sigma, \\
g(u)=\left(u+x^{*}\right) \rho\left(u+x^{*}\right)\left(-\gamma+\psi\left(u+x^{*}\right)\right)\left[\exp \left\{-\Phi\left(u+x^{*}\right)\right\}\right]^{2} .
\end{gathered}
$$

In fact, changing variables

$$
\begin{aligned}
u= & -x^{*} \\
v=- & (x \rho(x)-y \phi(x)) \exp \{-\Phi(x)\} \\
& +\int_{x^{*}}^{x}\left\{(-\gamma+\psi(\sigma))+\phi(\sigma) \frac{d}{d \sigma}\left(\frac{\sigma \rho(\sigma)}{\phi(\sigma)}\right)\right\} \exp \{-\Phi(\sigma)\} d \sigma, \\
d \tau=- & \exp \{\Phi(x)\} d t
\end{aligned}
$$

we have

$$
\begin{aligned}
\frac{d u}{d \tau} & =-(x \rho(x)-y \phi(x)) \exp \{-\Phi(x)\} \\
& =v-\int_{x^{*}}^{x}\left\{(-\gamma+\psi(\sigma))+\phi(\sigma) \frac{d}{d \sigma}\left(\frac{\sigma \rho(\sigma)}{\phi(\sigma)}\right)\right\} \exp \{-\Phi(\sigma)\} d \sigma \\
& =v-F(u)
\end{aligned}
$$


and

$$
\begin{aligned}
\frac{d v}{d \tau}=\{ & \left.\dot{x} \rho(x)+x \rho^{\prime}(x) \dot{x}-\dot{y} \phi(x)-y \phi^{\prime}(x) \dot{x}\right\}[\exp \{-\Phi(x)\}]^{2} \\
& -(x \rho(x)-y \phi(x)) \frac{\phi^{\prime}(x)}{\phi(x)} \dot{x}[\exp \{-\Phi(x)\}]^{2} \\
& \quad-\left\{(-\gamma+\psi(x))+\phi(x) \frac{d}{d x}\left(\frac{x \rho(x)}{\phi(x)}\right)\right\} \dot{x}[\exp \{-\Phi(x)\}]^{2} \\
= & \dot{x}\left\{\rho(x)+x \rho^{\prime}(x)-\frac{x \rho(x) \phi^{\prime}(x)}{\phi(x)}-\phi(x) \frac{d}{d x}\left(\frac{x \rho(x)}{\phi(x)}\right)\right\}[\exp \{-\Phi(x)\}]^{2} \\
& \quad-\{(-\gamma+\psi(x)) \dot{x}+\phi(x) \dot{y}\}[\exp \{-\Phi(x)\}]^{2} \\
=- & \{(-\gamma+\psi(x))(x \rho(x)-y \phi(x))+\phi(x) y(-\gamma+\psi(x))\}[\exp \{-\Phi(x)\}]^{2} \\
= & -x \rho(x)(-\gamma+\psi(x))[\exp \{-\Phi(x)\}]^{2} \\
= & -g(u) .
\end{aligned}
$$

The change of variables transfers the positive equilibrium $\left(x^{*}, y^{*}\right)$ of (1.2) to the origin of (2.1). It is clear that $F(0)=0$. By assumptions (i)-(iii) on $\rho(x), \phi(x)$, and $\psi(x)$ we see that

$$
u g(u)>0 \text { for }-x^{*}<u<K-x^{*} \text { and } u \neq 0 \text {. }
$$

Since

$$
F^{\prime}(u)=\left\{\left(-\gamma+\psi\left(u+x^{*}\right)\right)+\phi\left(u+x^{*}\right) \frac{d}{d u}\left(\frac{\left(u+x^{*}\right) \rho\left(u+x^{*}\right)}{\phi\left(u+x^{*}\right)}\right)\right\} \exp \left\{-\Phi\left(u+x^{*}\right)\right\}
$$

and

$$
\begin{aligned}
& g^{\prime}(u)=\rho\left(u+x^{*}\right)\left(-\gamma+\psi\left(u+x^{*}\right)\right)\left[\exp \left\{-\Phi\left(u+x^{*}\right)\right\}\right]^{2} \\
& +\left(u+x^{*}\right) \rho^{\prime}\left(u+x^{*}\right)\left(-\gamma+\psi\left(u+x^{*}\right)\right)\left[\exp \left\{-\Phi\left(u+x^{*}\right)\right\}\right]^{2} \\
& +\left(u+x^{*}\right) \rho\left(u+x^{*}\right) \psi^{\prime}\left(u+x^{*}\right)\left[\exp \left\{-\Phi\left(u+x^{*}\right)\right\}\right]^{2} \\
& \quad-2\left(u+x^{*}\right) \rho\left(u+x^{*}\right)\left(-\gamma+\psi\left(u+x^{*}\right)\right) \frac{\phi^{\prime}\left(u+x^{*}\right)}{\phi\left(u+x^{*}\right)}\left[\exp \left\{-\Phi\left(u+x^{*}\right)\right\}\right]^{2},
\end{aligned}
$$

we get

$$
F^{\prime}(0)=\left.\phi\left(x^{*}\right) \frac{d}{d u}\left(\frac{\left(u+x^{*}\right) \rho\left(u+x^{*}\right)}{\phi\left(u+x^{*}\right)}\right)\right|_{u=0} \quad \text { and } \quad g^{\prime}(0)=x^{*} \rho\left(x^{*}\right) \psi^{\prime}\left(x^{*}\right)>0 .
$$

Hence, by Corollary 2.1 we have the following result.

Theorem 2.2. System (1.2) has no homoclinic orbits. If

$$
\left.\frac{d}{d x}\left(\frac{x \rho(x)}{\phi(x)}\right)\right|_{x=x^{*}}=0
$$


then all positive semitrajectories of (1.2) near the positive equilibrium $\left(x^{*}, y^{*}\right)$ keep on rotating around $\left(x^{*}, y^{*}\right)$.

Since system (1.1) is a special case of the Gause predator-prey model (1.2) with $\gamma=D$, $K=k$,

$$
\rho(x)=r\left(1-\frac{x}{k}\right), \quad \phi(x)=\frac{x^{p}}{a+x^{p}}, \quad \text { and } \quad \psi(x)=\frac{\mu x^{p}}{a+x^{p}},
$$

the following is an immediate consequence of Theorem 2.2 .

THEOREM 2.3. System (1.1) has no homoclinic orbits. If

$$
(p D-(p-1) \mu) k=(p D-(p-2) \mu) \lambda_{p}
$$

then all positive semitrajectories of (1.1) near the positive equilibrium $E^{*}$ keep on rotating around $E^{*}$.

\section{NON-EXISTENCE OF CLOSED ORBITS}

In this section we will prove the following result concerning the non-existence of closed orbits of (1.1).

ThEOREM 3.1. Let $p$ be a positive number with $p<1$. If $(1.6)$ is satisfied, then system (1.1) has no closed orbits.

By a change of variables

$$
u=x-\lambda_{p}, \quad v=\log y-\log \nu_{p}, \quad d s=-\frac{x^{p}}{a+x^{p}} d t
$$

system (1.1) can be transformed into the system

$$
\begin{aligned}
& \frac{d u}{d s}=\nu_{p} e^{v}-r\left(1-\frac{u+\lambda_{p}}{k}\right)\left\{a\left(u+\lambda_{p}\right)^{1-p}+\left(u+\lambda_{p}\right)\right\} \\
& \frac{d v}{d s}=-\mu+D+a D\left(u+\lambda_{p}\right)^{-p}
\end{aligned}
$$

To pay our attention to the parameter $k$, we put

$$
\Gamma_{k}(u)=r\left(1-\frac{u+\lambda_{p}}{k}\right)\left\{a\left(u+\lambda_{p}\right)^{1-p}+\left(u+\lambda_{p}\right)\right\}-\nu_{p}
$$

for $u>-\lambda_{p}$. We also define

$$
\delta(u)=\mu-D-a D\left(u+\lambda_{p}\right)^{-p}
$$

for $u>-\lambda_{p}$. Then we have

$$
\begin{aligned}
& \frac{d u}{d s}=\nu_{p}\left(e^{v}-1\right)-\Gamma_{k}(u) \\
& \frac{d v}{d s}=-\delta(u)
\end{aligned}
$$


Since

$$
\Gamma_{k}(0)=r\left(1-\frac{\lambda_{p}}{k}\right) \lambda_{p}\left(\frac{a}{\lambda_{p}^{p}}+1\right)-\nu_{p}=0
$$

and

$$
u \delta(u)=a D u\left(\frac{1}{\lambda_{p}^{p}}-\frac{1}{\left(u+\lambda_{p}\right)^{p}}\right)>0 \quad \text { if } u \neq 0
$$

system (3.1) is of Liénard type.

Consider the plane curve $\left(\Gamma_{k}(u), \Delta(u)\right)$ for $u>-\lambda_{p}$, where

$$
\Delta(u)=\int_{0}^{u} \delta(\sigma) d \sigma
$$

This curve passes through the origin at $u=0$. The second component $\Delta(u)$ is decreasing for $-\lambda_{p}<u<0$ and increasing for $u>0$. Hence, the curve $\left(\Gamma_{k}(u), \Delta(u)\right)$ has a point of intersection with itself if and only if there exist two constants $u_{1}<0$ and $u_{2}>0$ such that

$$
\Gamma_{k}\left(u_{1}\right)=\Gamma_{k}\left(u_{2}\right) \quad \text { and } \quad \Delta\left(u_{1}\right)=\Delta\left(u_{2}\right) .
$$

It is known that if the curve $\left(\Gamma_{k}(u), \Delta(u)\right)$ has no point of intersection with itself, then system (1.1) has no closed orbits (and neither has system (1.1)). For the proof, we refer to [21]-[23].

Condition (1.6) yields

$$
k \leq \frac{p D-(p-2) \mu}{p D-(p-1) \mu} \lambda_{p} \equiv k^{*}
$$

when $0<p<1$. We intend to show that (1.6) implies the curve $\left(\Gamma_{k}(u), \Delta(u)\right)$ has no intersecting point with itself. To begin with, we examine a property of the curve $\left(\Gamma_{k^{*}}(u), \Delta(u)\right)$.

LEMMA 3.1. Let $H(u)$ be the inclination of the curve $\left(\Gamma_{k^{*}}(u), \Delta(u)\right)$, that is,

$$
H(u)=\frac{\Delta^{\prime}(u)}{\Gamma_{k^{*}}^{\prime}(u)}
$$

If $0<p<1$, then $H(u)<0$ and $H^{\prime}(u)>0$ for $u>-\lambda_{p}$ and $u \neq 0$.

Proof. Since

$$
\Gamma_{k^{*}}(u)=r\left(1-\frac{u+\lambda_{p}}{k^{*}}\right)\left\{a\left(u+\lambda_{p}\right)^{1-p}+\left(u+\lambda_{p}\right)\right\}-\nu_{p}
$$

and

$$
\Delta(u)=(\mu-D) u-\frac{a D}{1-p}\left\{\left(u+\lambda_{p}\right)^{1-p}-\lambda_{p}^{1-p}\right\}
$$

for $u>-\lambda_{p}$, we have

$$
\Gamma_{k^{*}}^{\prime}(u)=\frac{r}{k^{*}}\left\{\left(1+\frac{a(1-p)}{\left(u+\lambda_{p}\right)^{p}}\right) k^{*}-2\left(u+\lambda_{p}\right)-a(2-p)\left(u+\lambda_{p}\right)^{1-p}\right\}
$$




$$
\begin{gathered}
\Gamma_{k^{*}}^{\prime \prime}(u)=-\frac{r}{k^{*}}\left\{\frac{a k^{*} p(1-p)}{\left(u+\lambda_{p}\right)^{1+p}}+2+\frac{a(1-p)(2-p)}{\left(u+\lambda_{p}\right)^{p}}\right\}<0 \\
\Delta^{\prime}(u)=a D\left(\frac{1}{\lambda_{p}^{p}}-\frac{1}{\left(u+\lambda_{p}\right)^{p}}\right)
\end{gathered}
$$

and

$$
\Delta^{\prime \prime}(u)=\frac{a p D}{\left(u+\lambda_{p}\right)^{1+p}}>0
$$

for $u>-\lambda_{p}$. Using the fact that $a D=(\mu-D) \lambda_{p}^{p}$, we also have

$$
\begin{aligned}
\Gamma_{k^{*}}^{\prime}(0) & =\frac{r}{k^{*}}\left\{\left(1+\frac{a(1-p)}{\lambda_{p}^{p}}\right) k^{*}-2 \lambda_{p}-a(2-p) \lambda_{p}^{1-p}\right\} \\
& =\frac{r}{k^{*}}\left\{\left(1+\frac{(1-p)(\mu-D)}{D}\right) k^{*}-2 \lambda_{p}-\frac{(2-p)(\mu-D)}{D} \lambda_{p}\right\} \\
& =\frac{r}{k^{*} D}\left\{(p D+(1-p) \mu) k^{*}-(p D+(2-p) \mu) \lambda_{p}\right\}=0
\end{aligned}
$$

and

$$
\Delta^{\prime}(0)=a D\left(\frac{1}{\lambda_{p}^{p}}-\frac{1}{\lambda_{p}^{p}}\right)=0
$$

Hence, we see

$$
u \Gamma_{k^{*}}^{\prime}(u)<0 \text { and } u \Delta^{\prime}(u)>0 \text { if } u \neq 0
$$

Now, we consider the inclination

$$
H(u)=\frac{\Delta^{\prime}(u)}{\Gamma_{k^{*}}^{\prime}(u)}=\frac{a k^{*} D}{r \lambda_{p}^{p}}\left\{\frac{\left(u+\lambda_{p}\right)^{p}-\lambda_{p}^{p}}{\left(\left(u+\lambda_{p}\right)^{p}+a(1-p)\right) k^{*}-2\left(u+\lambda_{p}\right)^{1+p}-a(2-p)\left(u+\lambda_{p}\right)}\right\} .
$$

Since $\Gamma_{k^{*}}^{\prime}(0)=0$, the slope function $H(u)$ is not defined for $u=0$. From (3.2) it is clear that $H(u)<0$ for $u>-\lambda_{p}$ and $u \neq 0$. We also obtain

$$
\lim _{u \rightarrow-\lambda_{p}} H(u)=-\frac{D}{r(1-p)}, \quad \lim _{u \rightarrow \infty} H(u)=0, \quad \text { and } \quad \lim _{u \rightarrow 0} H(u)=\frac{\Delta^{\prime \prime}(0)}{\Gamma_{k^{*}}^{\prime \prime}(0)}<0 .
$$

We next show that

$$
H^{\prime}(u)=\frac{\Delta^{\prime \prime}(u) \Gamma_{k^{*}}^{\prime}(u)-\Delta^{\prime}(u) \Gamma_{k^{*}}^{\prime \prime}(u)}{\left\{\Gamma_{k^{*}}^{\prime}(u)\right\}^{2}}
$$

is positive for $u>-\lambda_{p}$ and $u \neq 0$. Since

$$
\Delta^{\prime \prime}(u) \Gamma_{k^{*}}^{\prime}(u)=\frac{a r D}{k^{*}}\left\{\frac{k^{*} p}{\left(u+\lambda_{p}\right)^{1+p}}+\frac{a k^{*} p(1-p)}{\left(u+\lambda_{p}\right)^{1+2 p}}-\frac{2 p}{\left(u+\lambda_{p}\right)^{p}}-\frac{a p(2-p)}{\left(u+\lambda_{p}\right)^{2 p}}\right\}
$$


and

$$
\begin{array}{r}
\Delta^{\prime}(u) \Gamma_{k^{*}}^{\prime \prime}(u)=-\frac{a r D}{k^{*}}\left\{\frac{a k^{*} p(1-p)}{\lambda_{p}^{p}\left(u+\lambda_{p}\right)^{1+p}}+\frac{2}{\lambda_{p}^{p}}+\frac{a(1-p)(2-p)}{\lambda_{p}^{p}\left(u+\lambda_{p}\right)^{p}}\right. \\
\left.-\frac{a k^{*} p(1-p)}{\left(u+\lambda_{p}\right)^{1+2 p}}-\frac{2}{\left(u+\lambda_{p}\right)^{p}}-\frac{a(1-p)(2-p)}{\left(u+\lambda_{p}\right)^{2 p}}\right\} \\
=-\frac{a r D}{k^{*}}\left\{\frac{k^{*} p(1-p)(\mu-D)}{D\left(u+\lambda_{p}\right)^{1+p}}+\frac{2}{\lambda_{p}^{p}}+\frac{(1-p)(2-p)(\mu-D)}{D\left(u+\lambda_{p}\right)^{p}}\right. \\
\left.-\frac{a k^{*} p(1-p)}{\left(u+\lambda_{p}\right)^{1+2 p}}-\frac{2}{\left(u+\lambda_{p}\right)^{p}}-\frac{a(1-p)(2-p)}{\left(u+\lambda_{p}\right)^{2 p}}\right\},
\end{array}
$$

we have

$$
\begin{aligned}
& \Delta^{\prime \prime}(u) \Gamma_{k^{*}}^{\prime}(u)-\Delta^{\prime}(u) \Gamma_{k^{*}}^{\prime \prime}(u)=\frac{a r D}{k^{*}}\left\{\frac{k^{*} p(p D+(1-p) \mu)}{D\left(u+\lambda_{p}\right)^{1+p}}+\frac{2}{\lambda_{p}^{p}}\right. \\
& \left.+\frac{(1-p)(2-p) \mu-\left(p^{2}-p+4\right) D}{D\left(u+\lambda_{p}\right)^{p}}-\frac{a(2-p)}{\left(u+\lambda_{p}\right)^{2 p}}\right\} \\
& =\frac{\operatorname{ar} D}{k^{*}}\left\{\frac{p(p D+(2-p) \mu) \lambda_{p}}{D\left(u+\lambda_{p}\right)^{1+p}}+\frac{2}{\lambda_{p}^{p}}\right. \\
& \left.+\frac{(1-p)(2-p) \mu-\left(p^{2}-p+4\right) D}{D\left(u+\lambda_{p}\right)^{p}}-\frac{a(2-p)}{\left(u+\lambda_{p}\right)^{2 p}}\right\} \\
& =\frac{a r W(u)}{k^{*}\left(u+\lambda_{p}\right)^{1+2 p}}
\end{aligned}
$$

where

$$
\begin{aligned}
W(u)=p(p D & +(2-p) \mu) \lambda_{p}\left(u+\lambda_{p}\right)^{p}+\frac{2 D}{\lambda_{p}^{p}}\left(u+\lambda_{p}\right)^{1+2 p} \\
& +\left((1-p)(2-p) \mu-\left(p^{2}-p+4\right) D\right)\left(u+\lambda_{p}\right)^{1+p}-a(2-p) D\left(u+\lambda_{p}\right) .
\end{aligned}
$$

Hence, the sign of $H^{\prime}(u)$ coincides with that of $W(u)$. We get

$$
\begin{aligned}
& W^{\prime}(u)=\frac{p^{2}(p D+(2-p) \mu) \lambda_{p}}{\left(u+\lambda_{p}\right)^{1-p}}+\frac{2(1+2 p) D}{\lambda_{p}^{p}}\left(u+\lambda_{p}\right)^{2 p} \\
& \quad+(1+p)\left((1-p)(2-p) \mu-\left(p^{2}-p+4\right) D\right)\left(u+\lambda_{p}\right)^{p}-a(2-p) D
\end{aligned}
$$

and

$$
\begin{array}{r}
W^{\prime \prime}(u)=\frac{1}{\left(u+\lambda_{p}\right)^{2}-p}\left\{-(1-p) p^{2}(p D+(2-p) \mu) \lambda_{p}+\frac{4 p(1+2 p) D}{\lambda_{p}^{p}}\left(u+\lambda_{p}\right)^{1+p}\right. \\
\left.+p(1+p)\left((1-p)(2-p) \mu-\left(p^{2}-p+4\right) D\right)\left(u+\lambda_{p}\right)\right\}
\end{array}
$$

We here define

$$
\begin{array}{r}
w(u)=-(1-p) p^{2}(p D+(2-p) \mu) \lambda_{p}+\frac{4 p(1+2 p) D}{\lambda_{p}^{p}}\left(u+\lambda_{p}\right)^{1+p} \\
+p(1+p)\left((1-p)(2-p) \mu-\left(p^{2}-p+4\right) D\right)\left(u+\lambda_{p}\right) .
\end{array}
$$


for $u>-\lambda_{p}$. Then we have

$$
w(0)=p(p(5-p) D+(1-p)(2-p) \mu) \lambda_{p}>0
$$

and

$$
\lim _{u \rightarrow-\lambda_{p}} w(u)=-(1-p) p^{2}(p D+(2-p) \mu) \lambda_{p}<0 .
$$

Also, we see that the function $w(u)$ is downwards convex. In fact, we have

$$
w^{\prime}(u)=\frac{4 p(1+p)(1+2 p) D}{\lambda_{p}^{p}}\left(u+\lambda_{p}\right)^{p}+p(1+p)\left((1-p)(2-p) \mu-\left(p^{2}-p+4\right) D\right)
$$

and

$$
w^{\prime \prime}(u)=\frac{4 p^{2}(1+p)(1+2 p) D}{\lambda_{p}^{p}}\left(u+\lambda_{p}\right)^{p-1}>0
$$

for $u>-\lambda_{p}$. Hence, there exists a $\hat{u}<0$ such that $w(\hat{u})=0$,

$$
w(u)<0 \quad \text { for }-\lambda_{p}<u<\hat{u} \quad \text { and } \quad w(u)>0 \quad \text { for } u>\hat{u} .
$$

Since $W^{\prime \prime}(u)=\frac{w(u)}{\left(u+\lambda_{p}\right)^{2-p}}$, the function $W^{\prime}(u)$ is decreasing for $-\lambda_{p}<u<0$ and increasing for $u>0$. Noticing that

$$
\begin{aligned}
\lim _{u \rightarrow 0} W(u)= & \lambda_{p}^{p}\left\{\left(p^{3}+2(1+2 p)-(1+p)\left(p^{2}-p+4\right)+(2-p)\right) D\right. \\
& \left.+\left(p^{2}(2-p)+(1+p)(1-p)(2-p)-(2-p)\right) \mu\right\} \\
=0 &
\end{aligned}
$$

and

$$
\lim _{u \rightarrow-\lambda_{p}} W^{\prime}(u)=\infty
$$

we conclude that $W^{\prime}(\bar{u})=0$ for some $\bar{u} \in\left(-\lambda_{p}, \hat{u}\right)$,

$$
W^{\prime}(u)>0 \quad \text { for }-\lambda_{p}<u<\bar{u} \text { or } u>0, \quad \text { and } \quad W^{\prime}(u)<0 \quad \text { for } \bar{u}<u<0 .
$$

Moreover, we can get

$$
W(0)=0 \quad \text { and } \quad \lim _{u \rightarrow-\lambda_{p}} W(u)=0 .
$$

Hence, it turns out that

$$
W(u)>0 \quad \text { for } u>-\lambda_{p} \text { and } u \neq 0 .
$$

Since the signs of $W(u)$ and $H^{\prime}(u)$ are the same, $H^{\prime}(u)$ is also positive for $u>-\lambda_{p}$ and $u \neq 0$.

The proof of Lemma 3.1 is now complete.

Remark 3.1. Let $\left(u_{1}, u_{2}\right)$ be a pair of constants satisfying

$$
-\lambda_{p}<u_{1}<0<u_{2} \text { and } \Delta\left(u_{1}\right)=\Delta\left(u_{2}\right) .
$$


Then it follows from Lemma 3.1 that $\Gamma_{k^{*}}\left(u_{2}\right)<\Gamma_{k^{*}}\left(u_{1}\right)$. That is, the curve $\left(\Gamma_{k^{*}}(u), \Delta(u)\right)$ has no point of intersection with itself (see Figure 1).

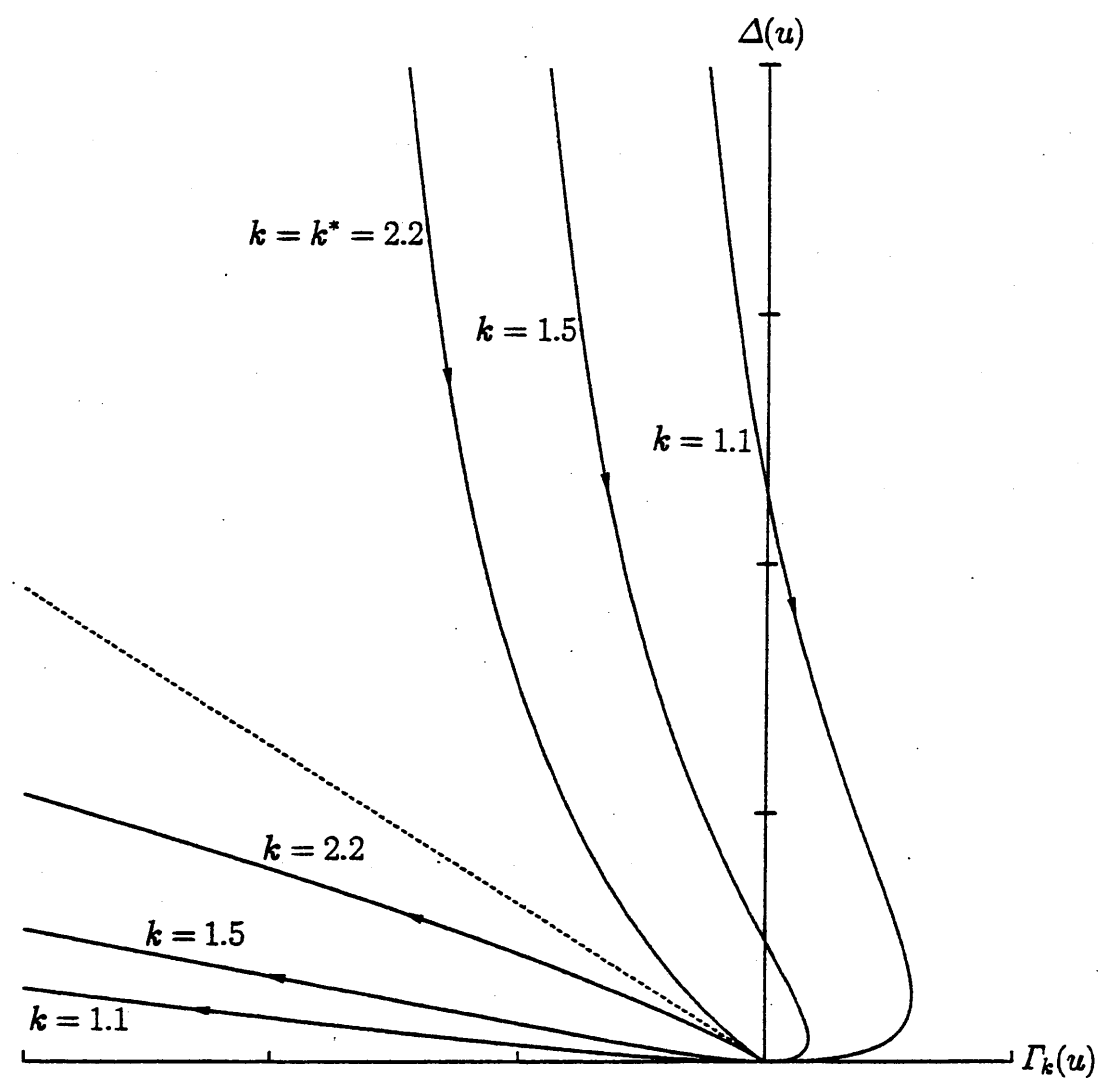

Fig. 1. The curve $\left(\Gamma_{k}(u), \Delta(u)\right)$ with $r=1, a=\frac{1}{3}, \mu=4, D=3, p=\frac{2}{3}$ and $k=$ $2.2,1.5,1.1$. The amount of $u$ increases in the direction of arrows.

Proof of Theorem 3.1. It is enough to show that the curve $\left(\Gamma_{k}(u), \Delta(u)\right)$ has no point of intersection with itself.

Partially differentiate $\Gamma_{k}(u)$ to obtain

$$
\frac{\partial}{\partial k} \Gamma_{k}(u)=\frac{r}{k^{2}}\left(a\left(u+\lambda_{p}\right)^{2-p}+\left(u+\lambda_{p}\right)^{2}-\frac{\mu}{D} \lambda_{p}^{2}\right) .
$$

Define

$$
f(u)=a\left(u+\lambda_{p}\right)^{2-p}+\left(u+\lambda_{p}\right)^{2}-\frac{\mu}{D} \lambda_{p}^{2}
$$

for $u>-\lambda_{p}$. Then we have

$$
f(0)=\lambda_{p}^{2}\left(\frac{\mu-D}{D}+1-\frac{\mu}{D}\right)=0
$$

and

$$
f^{\prime}(u)=a(2-p)\left(u+\lambda_{p}\right)^{1-p}+2\left(u+\lambda_{p}\right)>0 \text { for } u>-\lambda_{p}
$$


Hence, we get

$$
f(u)>0 \text { for } u>0 \text { and } f(u)<0 \text { for }-\lambda_{p}<u<0
$$

and, therefore,

$$
\frac{\partial}{\partial k} \Gamma_{k}(u)>0 \text { for } u>0 \text { and } \frac{\partial}{\partial k} \Gamma_{k}(u)<0 \text { for }-\lambda_{p}<u<0 .
$$

By (1.6) and the fact that $0<p<1$, the parameter $k$ is not greater than $k^{*}$. We therefore conclude that

$$
\Gamma_{k}(u)<\Gamma_{k^{*}}(u) \quad \text { for } u>0 \quad \text { and } \quad \Gamma_{k}(u)>\Gamma_{k^{*}}(u) \quad \text { for }-\lambda_{p}<u<0 \text {. }
$$

Thus, from Lemma 3.1 and Remark 3.1 it follows that

$$
\Gamma_{k}\left(u_{2}\right)<\Gamma_{k^{*}}\left(u_{2}\right)<\Gamma_{k^{*}}\left(u_{1}\right)<\Gamma_{k}\left(u_{1}\right)
$$

for any pair $\left(u_{1}, u_{2}\right)$ satisfying

$$
-\lambda_{p}<u_{1}<0<u_{2} \quad \text { and } \quad \Delta\left(u_{1}\right)=\Delta\left(u_{2}\right)
$$

This means that the curve $\left(\Gamma_{k}(u), \Delta(u)\right)$ has no intersecting point with itself (see Figure 1 again). The proof is complete.

It is clear that no closed orbits exist when asuumption (1.3) fails. Hence, combining Theorem 3.1 with Theorem A, we have

TheOREM 3.2. If (1.6) is satisfied, then system (1.1) has no closed orbits.

We are now ready to prove Theorem 1.1 which is the main result of this paper. In the next section, we give the proof of Theorem 1.1.

\section{PROOF OF THE MAIN RESULT}

Because of (1.3), system (1.1) has the unique positive equilibrium $E^{*}$. Taking the vector field into account, we can easily see that all solutions of (1.1) are positive and bounded in the future.

Sufficiency. Suppose that (1.6) is satisfied. We have to show that the positive equilibrium $E^{*}$ is stable and every solution of (1.1) tends to $E^{*}$.

Let $J^{*}$ be the variational matrix about $E^{*}$. Then we have

$$
J^{*}=\left(\begin{array}{cc}
M & -D / \mu \\
N & 0
\end{array}\right),
$$


where

$$
M=\frac{r}{k \mu}\left\{(p D-(p-1) \mu) k-(p D-(p-2) \mu) \lambda_{p}\right\}
$$

and

$$
N=\frac{p r}{k}\left(k-\lambda_{p}\right)(\mu-D)>0
$$

If

$$
(p D-(p-1) \mu) k<(p D-(p-2) \mu) \lambda_{p},
$$

then $M$ is negative and, therefore, the eigenvalues of $J^{*}$ have negative real parts. Hence, we see that the positive equilibrium $E^{*}$ is (locally asymptotically) stable.

In case

$$
(p D-(p-1) \mu) k=(p D-(p-2) \mu) \lambda_{p},
$$

form Theorem 2.3, all positive semitrajectory of (1.1) near the positive equilibrium $E^{*}$ keep on rotating arround $E^{*}$. Suppose that the positive equilibrium $E^{*}$ is not stable. Then every positive semitrajectory of (1.1) starting in the neighborhood of $E^{*}$ go away from $E^{*}$. Hence, by the uniqueness of solutions for the initial value problem and the Poincare-Bendixson theorem, system (1.1) has a closed orbit. This is a contradiction to Theorem 3.2. Thus, the positive equilibrium $E^{*}$ is also stable in the case.

From Theorem 3.2, system (1.1) has no closed orbits. Hence, by the Poincaré-Bendixson theorem again, we see that all positive semitrajectory approach the unique positive equilibrium $E^{*}$. That is, every solution of (1.1) tends to $E^{*}$.

Necessity. Supposse that

$$
(p D-(p-1) \mu) k>(p D-(p-2) \mu) \lambda_{p}
$$

namely, $M$ is positive. Then the eigenvalues of $J^{*}$ have positive real parts. Thus, the positive equilibrium $E^{*}$ is unstable.

We have completed the proof.

\section{DISCUSSION}

Consider the system

$$
\begin{aligned}
& \dot{x}=x \rho(x)-\xi(y) \phi(x), \\
& \dot{y}=\eta(y)(-\gamma+\psi(x)),
\end{aligned}
$$

where the functions $\rho, \xi, \phi, \eta, \psi$ are sufficiently smooth and the following assumptions:

$$
\begin{aligned}
& \text { there exists a } K>0 \text { such that }(x-K) \rho(x)<0 \text { if } x \neq K, \\
& \phi(0)=\psi(0)=0 \text { and } \phi^{\prime}(x)>0 \text { and } \psi^{\prime}(x)>0 \text { for } x>0, \\
& \xi(0)=\eta(0)=0 \text { and } \xi^{\prime}(y)>0 \text { and } \eta^{\prime}(y)>0 \text { for } y>0
\end{aligned}
$$


there exists an $x^{*}$ with $0<x^{*}<K$ such that $\psi\left(x^{*}\right)=\gamma$,

$$
\begin{aligned}
& \lim _{y \rightarrow \infty} \xi(y)>\frac{x^{*} \rho\left(x^{*}\right)}{\phi\left(x^{*}\right)}, \\
& \left.\frac{d}{d x}\left(\frac{x \rho(x)}{\phi(x)}\right)\right|_{x=x^{*}}<0 .
\end{aligned}
$$

Let $y^{*}$ be a positive constant satisfying

$$
\xi\left(y^{*}\right)=\frac{x^{*} \rho\left(x^{*}\right)}{\phi\left(x^{*}\right)} .
$$

Assumptions (5.2)-(5.6) guarantee that $\left(x^{*}, y^{*}\right)$ is a unique positive equilibrium and assumption (5.7) guarantees that $\left(x^{*}, y^{*}\right)$ is locally asymptotically stable.

Kuang [19] gave some sufficient conditions for the positive equilibrium $\left(x^{*}, y^{*}\right)$ of (5.1) to be globally asymptotically stable.

ThEOREM D ([19]). Assume (5.2)-(5.7). If one of the following conditions is satisfied, then the positive equilibrium $\left(x^{*}, y^{*}\right)$ of (5.1) is globally asymptotically stable:

$$
\begin{aligned}
& \qquad\left(\frac{x \rho(x)}{\phi(x)}-\xi\left(y^{*}\right)\right)\left(x-x^{*}\right) \leq 0 \quad \text { for } 0 \leq x \leq K ; \\
& \qquad \frac{d}{d x}\left(\frac{x \rho(x)}{\phi(x)}\right)<0 \text { for } 0 \leq x \leq K ; \\
& \phi(x) \frac{d}{d x}\left(\frac{x \rho(x)}{\phi(x)}\right)+\beta(-\gamma+\psi(x)) \leq 0 \text { for } 0 \leq x \leq K, \\
& \text { where } \beta \text { is a suitable positive constant; } \\
& \frac{d}{d x}\left(\frac{\tau(x)}{-\gamma+\psi(x)}\right) \geq 0 \text { for } 0<x<K \text { and } x \neq x^{*}, \\
& \text { where } \tau(x)=\phi(x) \frac{d}{d x}\left(\frac{x \rho(x)}{\phi(x)}\right) .
\end{aligned}
$$

Comparing system (1.1) with system (5.1), we see that $x^{*}=\lambda_{p}, y^{*}=\nu_{p}, \gamma=D, K=k$, $\xi(y)=\eta(y)=y$,

$$
\rho(x)=r\left(1-\frac{x}{k}\right), \quad \phi(x)=\frac{x^{p}}{a+x^{p}}, \quad \text { and } \quad \psi(x)=\frac{\mu x^{p}}{a+x^{p}} .
$$

Hence, we have

$$
\frac{x \rho(x)}{\phi(x)}-\xi\left(y^{*}\right)=\Gamma_{k}\left(x-\lambda_{p}\right) \quad \text { and } \quad-\gamma+\psi(x)=\phi(x) \delta\left(x-\lambda_{p}\right),
$$

where $\Gamma_{k}$ and $\delta$ are defined in Section 3 and, therefore, conditions (5.7)-(5.10) are equivalent to

$$
\begin{array}{ll} 
& \Gamma_{k}^{\prime}(0)<0 \\
u \Gamma_{k}(u) \leq 0 & \text { for }-\lambda_{p} \leq u \leq K-\lambda_{p},
\end{array}
$$




$$
\begin{gathered}
\Gamma_{k}^{\prime}(u)<0 \text { for }-\lambda_{p} \leq u \leq K-\lambda_{p} \\
\Gamma_{k}^{\prime}(u)+\beta \delta(u) \leq 0 \text { for }-\lambda_{p} \leq u \leq K-\lambda_{p} \\
\frac{d}{d u}\left(\frac{\delta(u)}{\Gamma_{k^{\prime}}^{\prime}(u)}\right) \leq 0 \text { for }-\lambda_{p} \leq u \leq K-\lambda_{p} \text { and } u \neq 0
\end{gathered}
$$

respectively.

Since $\Gamma_{k}(u)$ is a $C^{1}$-function and $\Gamma_{k}(0)=0$, condition $(5.8)^{\prime}$ implies $\Gamma_{k}^{\prime}(0) \leq 0$, that is,

$$
(p D-(p-1) \mu) k \leq(p D-(p-2) \mu) \lambda_{p}
$$

which is the necessary and sufficient condition for the global asymptotic stability of the equilibrium $E^{*}$ of (1.1). Of course, $(5.7)^{\prime}$ or $(5.9)^{\prime}$ implies $\Gamma_{k}^{\prime}(0) \leq 0$. Since $\delta(0)=0$, condition $(5.10)^{\prime}$ also implies $\Gamma_{k}^{\prime}(0) \leq 0$. Thus, condition (5.7) is somewhat heavy and conditions $(5.8)-(5,10)$ are unnecessary to ensure that the positive equilibrium $E^{*}$ of $(1.1)$ is globally asymptotically stable.

It was shown in the proof of Lemma 3.1 that

$$
\Gamma_{k^{*}}^{\prime}(0)=0
$$

and

$$
\frac{d}{d u}\left(\frac{\delta(u)}{\Gamma_{k^{*}}(u)}\right)=\frac{d}{d u}\left(\frac{\Delta^{\prime}(u)}{\Gamma_{k^{*}}(u)}\right)=H^{\prime}(u)>0 \quad \text { for } u>-\lambda_{p} \text { and } u \neq 0 .
$$

Hence, conditions (5.7), (5.9) and (5.11) are not satisfied in the critical case

$$
(p D-(p-1) \mu) k=(p D-(p-2) \mu) \lambda_{p}
$$

namely, $k=k^{*}$.

\section{REFERENCES}

1. Holling C. S., The components of predation as revealed by a study of small-mammal predation of the European pine sawfly, Can. Entomol. 91, 293-320 (1959).

2. Rosenzweig M. L. \& MacArthur R. H., Graphical representation and stability conditions of predator-prey interactions, Am. Nat. 97, 209-223 (1963).

3. Holling C. S., The functional response of predators to prey density and its role in mimicry and population regulation, Mem. Ent. Soc. Can. 45, 1-60 (1965).

4. Real L. A., Ecological determinants of functional response, Ecology 60, 481-485 (1972).

5. Kazarinoff N. D. \& van der Driessche P., A model predator-prey system with functional response, Math. Bioșci. 39, 125-134 (1978).

6. Cheng Kuoshung, Uniqueness of a limit cycle for a predator-prey system, SIAM J. math. Analysis 12, 541-548 (1981).

7. Chen Junping \& Zhang Hongde, The qualitative analysis of two species predator-prey model with Holling's type III functional response, Appl. Math. Mech. 7, 77-86 (1986).

8. Ding Sunhong; On a kind of predator-prey system, SIAM J. math. Analysis 20, 1426-1435 (1989). 
9. Sugie J., Miyamoto K. \& Morino K., Absence of limit cycles of a predator-prey system with a sigmoid functional response, Appl. Math. Lett. 9, 85-90 (1996).

10. Sugie J, Kohno R. \& Miyazaki R., On a predator-prey system of Holling type, Proc. Amer. Math. Soc. 125, 2041-2050 (1997).

11. May R. M., Stability and Complexity in Model Ecosystems, 2nd ed., Princeton Univ. Press, Princeton (1974).

12. Hsu Szebi, On global stability of a predator-prey system, Math. Biosci. 39, 1-10 (1978).

13. Freedman H. I., Deterministic Mathematical Models in Population Ecology, Marcel Dekker, New York (1980).

14. Cheng Kuoshung, Hsu Szebi \& Lin Songsun, Some results on global stability of a predator-prey system, J. Math. Biol. 12, 115-126 (1981).

15. Kuang Yang \& Freedman H. I., Uniqueness of limit cycles in Gause-type models of predator-prey systems, Math. Biosci. 88, 67-84 (1988).

16. Ardito A. \& Ricciardi P., Lyapunov functions for a generalized Gause-type model, J. Math. Biol. 33, 816-828 (1995).

17. Kooij R. E. \& Zegeling A., Qualitative properties of two-dimensional predator-prey systems, Nonlinear Analysis 29, 693-715 (1997).

18. Gause G. F., The Struggle for Existence, Williams and Wilkins, Baltimore (1934).

19. Kuang Yang, Global stability of Gause-type predator-prey systems, J. Math. Biol. 28, 463-474 (1990).

20. Sugie J. \& Hara T., Existence and non-existence of homoclinic trajectories of the Liénard system, Disc. Conti. Dynam. Syst. 2, 237-254 (1996).

21. Cherkas L. A., Estimation of the number of limit cycles of autonomous systems, Differential Equations 13, 529-547 (1977).

22. Sugie J. \& Hara T., Non-existence of periodic solutions of the Liénard system, J. math. Analysis. Applic. 159, 224-236 (1991).

23. Gasull A. \& Guillamon A., Non-existence of limit cycles for some predator-prey systems, In Proceedings of equadiff'91, pp. 538-546, World Scientific, Singapore (1993). 